\title{
A Portable Dual Energy X-ray Absorptiometry Technique for the Measurement of Bone Mineral in Preterm Infants
}

\author{
J. R. WILLIAMS, F. DAVIDSON, G. MENON, AND N. MCINTOSH \\ Departments of Medical Physics and Medical Engineering |J.R.W., F.D./ and Child Life and \\ Health [G.M., N.M.|, University of Edinburgh, Edinburgh EH4 $2 X U$, United Kingdom
}

\begin{abstract}
A dual energy $x$-ray absorptiometry technique has been developed for the measurement of bone mineral in preterm infants. Two films are taken of the infant's forearm using a standard mobile $x$-ray set on the ward at two $x$-ray energies. Included in the field of view is a double step wedge made of aluminum and Perspex. The images are digitized by viewing them on a light box with a TV camera. The bone mineral content in the forearm can then be mapped by the measurement of light intensity in matching pixels of the two films and comparison with the intensities in the step wedge. The bone mineral concentration in the radius, expressed as bone mineral per unit length $(\mathrm{mg} / \mathrm{mm})$, is calculated. The technique has been tested using phantoms of calcium hydroxylapatite set in a tissue equivalent material and in Perspex. It has been shown that the reproducibility
\end{abstract}

ABSTRACT

of the technique is approximately $7 \%$ and that there is a systematic underestimate of bone mineral of about $17 \%$. The technique has been shown to be linear up to a bone mineral concentration of $8 \mathrm{mg} \cdot \mathrm{mm}^{-1}$. Longitudinal studies for 14 infants that show an initial 6-wk period of demineralization after birth with a subsequent mineralization phase are reported. (Pediatr Res 36: 351-357, 1994)
Abbreviations
BM, total mass of bone mineral
BMC, bone mineral concentration equal to mass of bone mineral divided by radius length
CR, computed radiography
$\boldsymbol{\mu}(\mathrm{E}) / \rho$, mass attenuation coefficient at energy $\mathrm{E}$

A number of techniques have been described for the measurement of bone mineral content using photon absorptiometry $(1,2)$. One of the problems of such measurements for preterm infants is the stress to the infant from being transported to the measuring device and from the handling required for the procedure. Previous studies have inevitably been restricted to infrequent measurements on relatively well infants (3). Mineral deposition is likely to be particularly compromised in the sick preterm infant. It would therefore be useful to have a system that could be used on the neonatal ward with minimal disturbance to the infant.

One approach to this problem was briefly outlined by Hawkes et al. (4) with preliminary clinical results presented by Lyon et al. (5). The technique uses radiographic images of the forearm taken with the mobile $\mathrm{x}$-ray unit used routinely in the neonatal unit. Two radiographs are taken at different energies (40 and $120 \mathrm{kV}$ ), and by

Received April 6, 1993; accepted March 21, 1994.

Correspondence and reprint requests: J. R. Williams, Department of Medical Physics and Medical Engineering. Western General Hospital, Edinburgh EH4 2XU, United Kingdom.

Supported by Milupa and a grant from the Scottish Office Home and Health Department. means of a double step wedge included within the field of view, the digitized images may be analyzed to yield the bone mineral content of the forearm.

The technique we have used was originally developed by Hawkes et al. (4) but has not been fully described by them. In this paper, we have therefore briefly summarized the theory behind the method. The implementation of the technique at this center is described, including the design of an arm holder that results in minimal disturbance to the baby. Developments in the technique include corrections for image nonuniformity and for movement. The results of measurements on phantoms to test accuracy and precision are reported. The technique has been used for clinical studies that will be the subject of future publications. However, to demonstrate that it can be used in practice for longitudinal studies of very-low-birth-weight infants, we present some preliminary clinical data.

\section{METHODS}

\section{Theory}

It has been shown (6) that the mass attenuation coefficient of one material ( $x$ ) may be related to the mass 
attenuation coefficients of two other materials ( $y$ and $z$ ) by a linear relationship of the form:

$$
[\mu(E) / \rho]_{x}=\alpha_{x y}[\mu(E) / \rho]_{y}+\alpha_{x z}[\mu(E) / \rho]_{z}
$$

The coefficients, $\alpha_{\mathrm{xy}}$ and $\alpha_{\mathrm{xz}}$, are constant provided that the photon energy, $\mathrm{E}$, is several times greater than the K-edge energy of the materials involved.

An $\mathrm{x}$-ray image is a map of the transmission through the overlying tissues. If it is assumed that there are two tissue components, bone mineral and water, and that the thickness of tissue is such that beam hardening is not significant, then:

$$
\ln \left(\mathrm{I} / \mathrm{I}_{0}\right)=-\mathrm{m}_{\mathrm{b}}[\mu(\mathrm{E}) / \rho]_{\mathrm{b}}-\mathrm{m}_{\mathrm{w}}[\mu(\mathrm{E}) / \rho]_{\mathrm{w}}
$$

in which $1 / I_{0}$ is the transmitted fraction of the $x$-ray beam and $m_{b}$ and $m_{w}$ are the thicknesses of bone mineral and water expressed as mass per unit area.

Using equation 2, the transmission through the two tissue components can be equated to the transmission through a mix of two reference materials, which in this work are aluminum and Perspex:

$$
m_{a}[\mu(E) / \rho]_{a}+m_{p}[\mu(E) / \rho]_{p}=m_{b}[\mu(E) / \rho]_{b}+m_{w}[\mu(E) / \rho]_{w}
$$

The subscripts $a, p, b$, and $w$ refer to aluminum, Perspex, bone mineral, and water, respectively.

The mass attenuation coefficients of aluminum and Perspex are related to those of bone mineral and water by relationships of the type shown in equation 1 . It is possible to show that equation 3 may be rewritten as:

$$
\begin{aligned}
\left(m_{b}-m_{a} \alpha_{a b}-\right. & \left.m_{p} \alpha_{p b}\right)[\mu(E) / \rho]_{b} \\
& =-\left(m_{w}-m_{a} \alpha_{a w}-m_{p} \alpha_{p w}\right)[\mu(E) / \rho]_{w}
\end{aligned}
$$

Provided that the coefficients $\alpha_{\mathrm{ab}}$, etc. are independent of energy over the range of interest, it can be seen that for a particular combination of bone mineral and water thicknesses there is a unique combination of aluminum and Perspex that would give the same attenuation over that energy range. These thicknesses are related by:

$$
m_{b}=m_{a} \alpha_{a b}+m_{p} \alpha_{p b}
$$

and

$$
\mathrm{m}_{\mathrm{w}}=\mathrm{m}_{\mathrm{a}} \alpha_{\mathrm{aw}}+\mathrm{m}_{\mathrm{p}} \alpha_{\mathrm{pw}}
$$

The technique used in this work is concerned with the derivation of $m_{a}$ and $m_{p}$ within each image pixel of two radiographs of the subject's forearm taken at two $x$-ray energies. Equation 5 can then be used to calculate bone mineral mass. To achieve this, a double step wedge made of Perspex and aluminum is included within the field of view (Fig. 1).

Values of the coefficients in equations 5 and 6 were derived from the data of Hubbell (7). Bone mineral was assumed to be calcium hydroxylapatite $\left[\mathrm{Ca}_{10}\left(\mathrm{PO}_{4}\right)_{6}(\mathrm{OH})_{2}\right]$, which was the material used to check the calibration of the
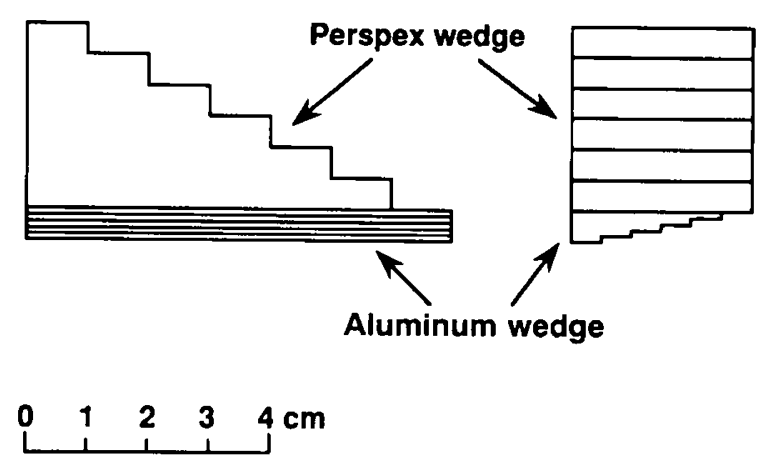

Figure 1. Cross-sectional views of the Perspex and aluminum wedge used for calibration.

technique. The radiographs were taken at 40 and $120 \mathrm{kV}$ so that the photon energy range for which the coefficients should apply is approximately 15 to $120 \mathrm{keV}$, the minimum and maximum photon energies in the two spectra, respectively (8). The tabulated values of mass attenuation coefficient from Hubbell (7) were interpolated for 25 and $70 \mathrm{keV}$ and the coefficients of the type shown in equation 1 for bone mineral and water were calculated at these energies. These values are $\alpha_{\mathrm{ab}}=0.4592, \alpha_{\mathrm{aw}}=0.4485, \alpha_{\mathrm{pb}}=-0.03637$, and $\alpha_{p w}=1.0051$.

To test the validity of equation 1 , the mass attenuation coefficients for the reference materials, aluminum and Perspex, were calculated from those of bone mineral and water. These values were compared with Hubbell's data over the energy range 10 to $150 \mathrm{keV}$ (Fig. 2). It can be seen that at $20 \mathrm{keV}$ there is a discrepancy of $1.7 \%$ for aluminum that decreases at higher energies.

The method of deriving bone mineral can be illustrated by considering the OD of the corresponding point in the forearm for the two x-ray films taken at 40 and $120 \mathrm{kV}$. For each radiograph, the OD of the chosen point or pixel can be compared with the densities in the Perspex/ aluminum step wedge. By interpolation between the steps of the wedge, a curve of equal OD can be plotted as a function of the thicknesses of the two wedge materials. This is illustrated in Figure 3, in which the two lines are drawn for the OD of corresponding pixels in the low- and high-kV radiographs. Each curve represents the combi-

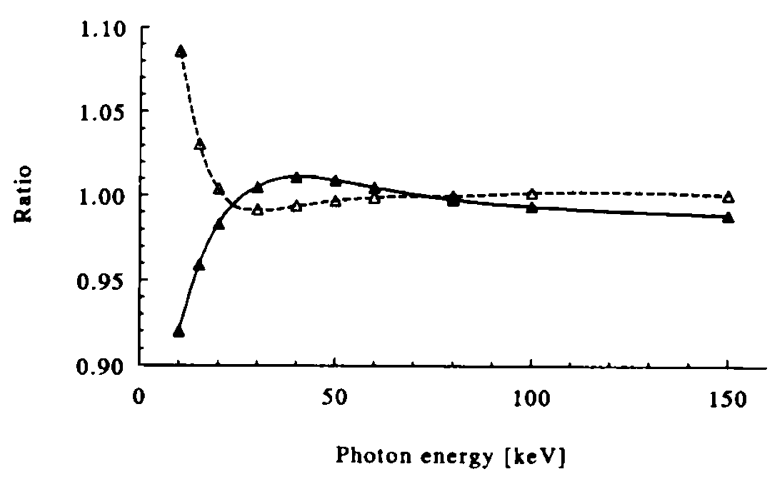

Figure 2. The ratio of mass attenuation coefficient for aluminum (dashed line) and Perspex (solid line) calculated from equations 5 and 6 to the values tabulated in reference 7 plotted against photon energy. 
Perspex thickness [mm]

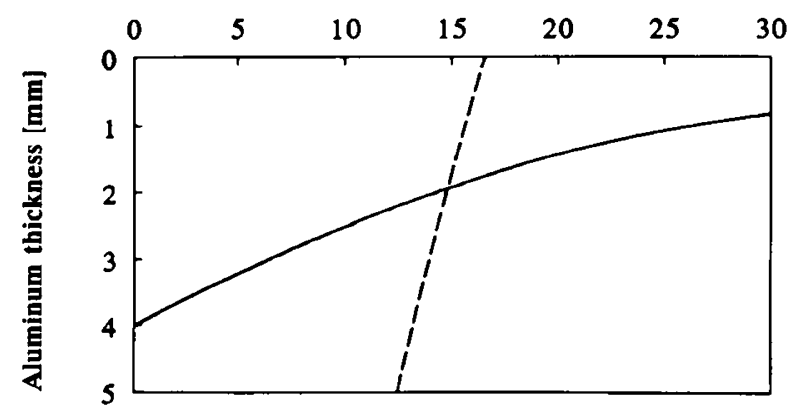

Figure 3. Lines of equal OD in the $x$-ray image of the calibration wedge plotted against aluminum and Perspex thickness. The solid line is for the low-kV $\mathrm{x}$-ray film and the dashed line for high-kV film.

nations of aluminum and Perspex thicknesses that would give the same $O D$ as the pixel of interest within the forearm. Thus, the intersection of the two lines represents a unique combination of aluminum and Perspex thicknesses that would produce the same OD as measured below the pixel of interest in the forearm. These values may then be used to calculate the mass of bone mineral and water overlying that pixel using equations 5 and 6. Qualitatively, this illustrates the method whereby a map of the bone mineral content of the subject's forearm may be derived.

\section{Measurement Technique}

The baby's forearm is held in position using a specially designed holder, which is shown in Figure 4. It has a thin polyethylene strap that can be tightened over the arm. Once the arm is positioned, the holder is placed on the film cassette.

The holder incorporates the aluminum/Perspex step wedge (Fig. 1). It is constructed of six 5-mm steps of Perspex and five 1-mm steps of aluminum. The aluminum used in the construction of the wedge was $99.999 \%$ pure (Goodfellow Metals Ltd., Cambridge, UK).

The radiographs were taken with an Explorer mobile $\mathbf{x}$-ray unit (Picker International, Inc., Cleveland, $\mathrm{OH}$ )

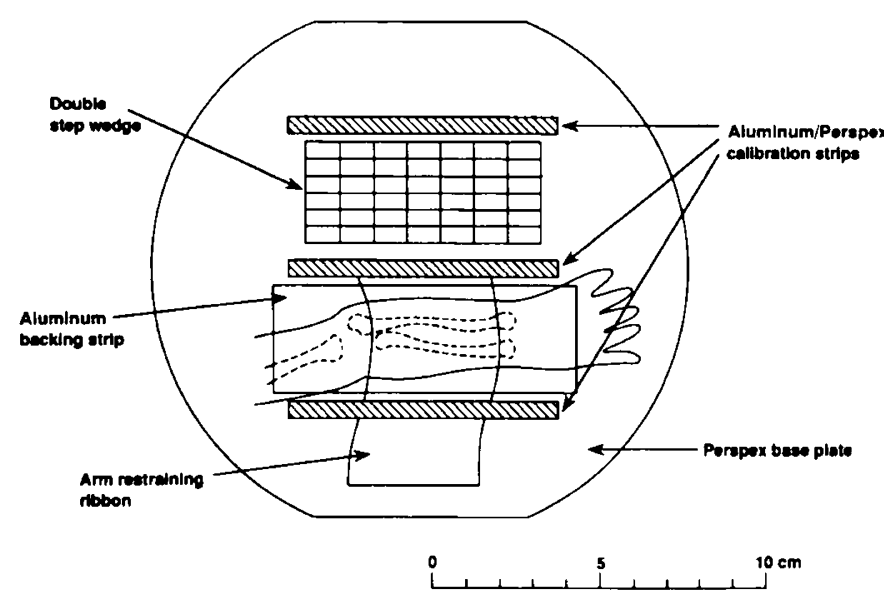

Figure 4. The holder used to position the infant's arm, showing the position of the step wedge and calibration strips. using $13 \times 18-\mathrm{cm}$ film cassettes incorporating type $\mathrm{T} 2$ intensifying screens $(3 \mathrm{M}$, St. Paul, $\mathrm{MN})$. The first radiograph was taken at $40 \mathrm{kV}$ and $6.3 \mathrm{~mA}$. The first cassette was then removed and replaced by a second one, with care taken to cause the minimum possible disturbance to the forearm position. A second exposure at $120 \mathrm{kV}$ and $3.2 \mathrm{~mA}$ was made. For the high-kV exposure, it was necessary to reduce the output of the $x$-ray set. This was achieved using a 4-mm copper filter. A secondary effect of this filter is to produce additional beam hardening. Although not quantified, this may be beneficial because of the consequent reduction in the proportion of attenuation due to the photoelectric effect. This would result in a reduction in the slope of the high- $k V$ curve of equal OD in Figure 3. The resultant increase in the angle between the curves may allow a more precise measurement of the point of intersection and thus of bone mineral.

Absorbed dose was measured at the position of the arm in the holder using a Radcal 2025 radiation monitor with a model 20X5-3 ionization chamber (Radcal Corp., Monrovia, CA). The measured doses were 90 and $20 \mu \mathrm{Gy}$ for the low- and high-kV settings, respectively, and represent the absorbed dose to the skin at the entrance surface. The absorbed doses due to scatter at a distance of 4 $\mathrm{cm}$ from the edge of the beam were measured with a model 20X5-180 chamber. The doses were 1 and $0.5 \mu \mathrm{Gy}$ for the two energies. The distance is approximately equal to the closest part of the head and trunk of the baby to the beam, and the dose at this distance serves as an upper limit estimate for the effective dose equivalent for one pair of radiographs, that is $1.5 \mu \mathrm{Sv}$.

The arm holder and film cassettes are sufficiently small to be introduced through the porthole of the incubator. In practice, this is important because it eliminates the need to open the incubator fully, which can cause unnecessary disturbance to the very sick patient. The studies can be done with the infant either prone or supine.

After processing, the films were digitized by viewing them on a light box using a charge-coupled device TV camera (Sony model XC77CE). The video signal was fed to a frame grabber as input to a MicroVax II computer (Digital Equipment Corp., Marlboro, MA). The position of the second film to be digitized was matched to the first using the image overlay facility of the frame grabber to match certain high-contrast details that could be seen in the image of the holder. On the digitized images, the operator marked the position of the wedge. The average relative light intensities associated with each of the 42 steps were then calculated for each film. Two $256 \times 256$ matrices of light intensity as a function of aluminum and Perspex thicknesses were interpolated for each energy. These matrices were then inverted to provide $256 \times 256$ look-up tables for each of the reference materials, giving thickness of the material as a function of low- and high-kV image light intensity. Thus, the light intensity in matching pixels in the two films could be interpreted as equivalent thicknesses of aluminum and Perspex from which the mass of bone mineral and water could be 
calculated using equations 5 and 6 . In this way, images of equivalent bone mineral and water mass were generated with the pixel value corresponding to mass per pixel.

The bone mineral content in the radius was then derived from these images. An outline was drawn around the bone with a margin of about 1 to $2 \mathrm{~mm}$. Profiles, one pixel in width, were generated approximately perpendicular to the bone, and these were integrated between the limits of the outlined region. A background equal to the average value of the two pixels adjacent to each side of the integrated area were then subtracted to give bone mineral within that section of the bone. This integration was repeated over the bone length to give the BM in the radius. This was divided by the length of the radius measured by ruler directly from the low-kV $\mathrm{x}$-ray image to give $\mathrm{BMC}$.

\section{Practical Implementation of Technique}

In practice, various factors need to be taken into account when applying the technique described above.

Light box nonuniformity. The light intensity of the viewing box was not uniform even over the relatively small area of film used in these measurements. This was readily corrected using films with a homogeneous OD over the area of interest. The light intensity measured in each pixel of the test film was multiplied by the ratio of the average intensity in the field of view of the uniformity film to the intensity of the corresponding pixel in that film. This also corrected for any nonuniformity in the response of the TV camera.

$X$-ray image nonuniformity. The $\mathrm{x}$-ray image of a homogeneous phantom would be nonuniform for a number of different reasons. These include variations in primary and secondary $x$-ray intensities, film and intensifying screen heterogeneity, and processor effects. These effects vary in degree from film to film, and in practice they cannot be eliminated or made consistent. Therefore, a method was devised to correct each film separately.

Three uniformity correction strips were incorporated into the arm holder. These were 1.2-mm-thick strips of pure aluminum beneath $10 \mathrm{~mm}$ of Perspex that lie parallel to the forearm, one between the arm and the wedge and two close to the edge of the irradiated area. The positions can be seen in the diagram of the arm holder (Fig. 4). After digitization and correction for light box nonuniformity, the images were converted to OD. This can be achieved through a comparison with the light intensities measured for the homogeneous films that were used for the light box nonuniformity correction. The difference between the OD at a particular pixel in the test film $\left(\tau_{\mathrm{i}, \mathrm{j}}\right)$ and the density of the uniformity film $\tau(u)$ is given by:

$$
\tau_{i, j}-\tau(u)=\log _{10}\left[I(u)_{i, j} / I_{i, j}\right]
$$

in which $\mathrm{I}(\mathrm{u})_{\mathrm{i}, \mathrm{j}}$ is the measured light intensity transmitted through the uniformity film at the position of the $(i, j)$ pixel and $I_{i, j}$ is the corresponding intensity measured through the test film. In the correction for light box nonuniformity, the transmitted intensity in the test film was corrected for the measured variations in intensity across the uniformity film. Thus, the equation can be rewritten to give:

$$
\tau_{\mathrm{i}, \mathrm{j}}=\log _{10}\left[\overline{\mathrm{I}}(\mathrm{u}) / \mathrm{I}_{\mathrm{i}, \mathrm{j}}^{\prime}\right]+\tau(\mathrm{u})
$$

in which $\bar{I}(u)$ is the average transmitted intensity through the uniformity film and $\mathrm{I}_{\mathrm{i}, \mathrm{j}}^{\mathrm{j}}$ is the measured intensity for the test film after correction for light box nonuniformity.

The OD profiles along the uniformity strips were calculated and corrections to the image pixel values between the uniformity strips were made by assuming a linear correction after subtraction of the background film fog. After this correction, the OD map was normalized to the central position of the middle strip before recalculation as a conventional light intensity image.

Correction for movement. There is inevitably some movement between the two radiographs, and two strategies were adopted to identify the degree of movement and to make corrections. Before the radiographs were taken, two $1.5-\mathrm{mm}$ lead markers were taped to the skin, one on the back of the hand and the second close to the elbow. Before analysis, the film pair were overlaid on a light box and matched according to the image of the holder. If the lead markers' positions were then found to coincide, this indicated that any movement between the radiographs was unlikely and no further correction was required. A mismatch of the markers showed that there had been some movement. Although a useful indicator, the markers could not be used to align the images because it was found they could move relative to the radius.

Although the contrast in the high-kV image is very poor, it is possible to discern some of the bone/soft tissue edge as well as the boundary of the arm. By tracing these edges from the high-kV film and by superimposing them over the low-kV image, matching of the two images could be achieved. After the uniformity corrections above, the image of the arm in the high- $\mathrm{kV}$ film was rotated or translated to fit the low-kV image.

\section{Validation of Technique}

A number of phantom and other studies have been performed to test the experimental methods. These have been concerned with reproducibility, linearity, absolute accuracy, and the effect of overlying soft tissue.

Reproducibility. Reproducibility has been tested with a phantom made of the tissue-equivalent material Mix-D (9). This was circular in cross section, with its upper and lower surfaces flattened to give a thickness of $20 \mathrm{~mm}$. It was constructed in two halves with a slot cut in the lower half that was filled with calcium hydroxylapatite held in place with a resin-based glue. The mass of hydroxylapatite was $188 \mathrm{mg}$ in a $40 \times 3.5-\mathrm{mm}$ slot that is typical of the size of the radius for these investigations. Four sets of radiographs were taken of this phantom over a period of $2 \mathrm{y}$. The sets comprised seven or six pairs of high- and 
low-kV images. The first set was analyzed on three occasions and the other sets once.

These tests were used to assess the coefficient of variance of the technique and to indicate whether there was any changes in sensitivity over the time period of the clinical studies. Variations in results may arise both from factors affecting the quality of the individual radiographs and from the digitization and computer analysis of the films. To test the latter effects, the analysis of one of the pairs of clinical films was repeated in full five times. In addition, certain parts of the analysis were repeated to assess their effects. Outlining of the region of interest for two pairs of clinical films was tested as well as the effect of repeating the alignment correction.

Linearity. To test linearity, four phantoms were constructed. These were rectangular blocks of Perspex 70 $\mathrm{mm}$ long and $24 \mathrm{~mm}$ wide. They were made in two halves with a $40 \times 4$-mm slot cut in one half. The depth of the slot depended on the amount of calcium hydroxylapatite to be added and varied between $1 \mathrm{~mm}$ and $3 \mathrm{~mm}$. The overall thickness of the phantoms differed so that the total Perspex thickness overlying the hydroxylapatite was $16 \mathrm{~mm}$ in all cases. The phantoms were filled with weighed amounts of calcium hydroxylapatite that varied between 94.7 and $322.7 \mathrm{mg}$. A fifth phantom was constructed that contained no hydroxylapatite. Three pairs of high-and low-kV films were obtained for each of these phantoms.

Effect of overlying soft tissue. To assess the effect of the thickness of overlying tissue on bone mineral measurement, an additional phantom was made. It was similar to the Perspex/hydroxylapatite phantoms used for the linearity tests except that the overall thickness of Perspex overlying the hydroxylapatite was $5.5 \mathrm{~mm}$. This was used in conjunction with additional blocks of Perspex to increase the overall thickness of Perspex in 5-mm steps up to $25.5 \mathrm{~mm}$. The mass of hydroxylapatite in this phantom was $169.0 \mathrm{mg}$. Three separate sets of films were taken and analyzed for each phantom thickness.

Accuracy. The phantom films described above were used to assess the absolute accuracy of the technique by comparison of the measured bone mineral with the weighed quantities of calcium hydroxylapatite.

\section{Clinical Films}

The technique has been developed for longitudinal studies of preterm infants. These studies have involved weekly measurements of bone mineral from birth until discharge from the unit. The technique has been used to study the effect of different types of feed on bone mineral. This work will be the subject of a separate report but, for illustration, data are presented here from 14 infants who were fed their own mother's breast milk supplemented with preterm formula. These subjects all had six or more analyzable film pairs and had been studied over a period of 7 wk or longer. The study from which these data were taken was approved by the hospital's ethical committee and required informed consent from the parents.

\section{RESULTS}

\section{Phantom Studies}

Reproducibility. The results of the phantom reproducibility tests are shown in Tables 1 and 2. Table 1 shows that the analysis program introduces random uncertainties of between approximately $1 \%$ and $2 \%$. Larger coefficients of variation (up to $9 \%$ ) were found for repeat $x$-ray films of the same phantom (Table 2). The coefficient of variation varied between $6.0 \%$ and $9.2 \%$ for these measurements, except for one set for which it was $1.7 \%$. The overall reproducibility is the average of these coefficients of variation calculated as their root mean square, which is $6.5 \%$.

The mean values of bone mineral for the four sets of films varied between $145.4 \pm 7.8$ and $158.6 \pm 6.4$. The uncertainties represent twice the SEM.

Linearity. The results of the linearity test are shown in Figure 5. It will be seen that the results fit a linear relationship. If the line is constrained to pass through the origin, its slope is given by 0.844 with a standard error of 0.010 .

Effect of overlying tissue. The results of these tests are shown in Table 3 . It can be seen that there is no significant change in the total bone mineral content in the phantom when the thickness of Perspex overlying the hydroxylapatite is increased from 5.5 to $20.5 \mathrm{~mm}$, that is for the first four of the five measurements. The average bone mineral content for these was $140.6 \pm 7.7 \mathrm{mg}$.

There was an apparent reduction of about $10 \%$ in the measured value of bone mineral when the total thickness of Perspex was increased to $25.5 \mathrm{~mm}$. This is equivalent to approximately $30 \mathrm{~mm}$ of soft tissue. For these latter measurements, it was noted that the fluctuations in the pixel values within the region of interest were such that the calculated value of bone mineral in certain pixels was falling below zero. This indicates that the measurements were being made close to the limit of tissue thickness that

Table 1. Reproducibility tests: repeat analysis of the same film pairs giving the BM in the phantom

\begin{tabular}{clcccc}
$\begin{array}{c}\text { Sub- } \\
\text { analysis* }\end{array}$ & Film $\dagger$ & $\begin{array}{c}\text { Run } \\
\text { by } \ddagger\end{array}$ & $\begin{array}{c}\text { No. of } \\
\text { repeats }\end{array}$ & $\begin{array}{c}\text { BM } \\
(\mathrm{mg})\end{array}$ & $\begin{array}{c}\text { Coefficient of } \\
\text { variance (\%) }\end{array}$ \\
\hline 1 & MD/CaH & FD & 5 & 152.2 & 1.8 \\
2 & SN-213 & FD & 5 & 115.6 & 1.0 \\
3 & SN-15 & JRW & 6 & 130.4 & 1.5 \\
& & FD & 5 & 129.5 & 1.8 \\
& SN-213 & JRW & 7 & 115.6 & 2.2 \\
& & FD & 5 & 115.8 & 0.9 \\
\hline
\end{tabular}

* Subanalyses: 1, full analysis of each film including digitization; 2 , analysis excluding digitization and uniformity correction; and 3, region of interest selection alone.

† Film: $\mathrm{MD} / \mathrm{CaH}, \mathrm{Mix}-\mathrm{D} /$ calcium hydroxylapatite phantom film pair from set $A$ used in part $A$ of reproducibility tests; $\mathrm{SN}-15$ and $\mathrm{SN}-213$, two pairs of clinical films.

$\ddagger$ Letters are the initials of the investigator conducting the test. 
Table 2. Reproducibility tests: repeat films of Mix-D/calcium hydroxylapatite phantom to show the variation in the measured BM

\begin{tabular}{ccrccc}
\hline $\begin{array}{c}\text { Film } \\
\text { set }\end{array}$ & $\begin{array}{c}\text { No. of } \\
\text { films }\end{array}$ & Date & $\begin{array}{c}\text { Run } \\
\text { by* }\end{array}$ & $\begin{array}{c}\text { BM } \\
(\mathrm{mg})\end{array}$ & $\begin{array}{c}\text { Coefficient of } \\
\text { variance }(\%)\end{array}$ \\
\hline A & 7 & $1 / 91$ & JRW & $143.6 \dagger$ & 8.2 \\
A & 7 & $5 / 91$ & JRW & 159.5 & 9.2 \\
A & 7 & $10 / 91$ & FD & 157.8 & 6.2 \\
B & 6 & $10 / 92$ & FD & 151.0 & 1.7 \\
C & 6 & $1 / 93$ & FD & 145.4 & 6.6 \\
D & 6 & $2 / 93$ & FD & 157.2 & 6.0 \\
\hline
\end{tabular}

* Letters are the initials of the investigator.

$\dagger$ Between January and May 1991, an error in the analysis program that had caused results to be low by a constant factor was corrected. The value of $\mathrm{BM}$ was therefore incorrect for this measurement.

can be handled by the method. This maximum thickness is related to the thickness of the calibration wedge.

Accuracy. The weighed mass of hydroxylapatite in the Mix-D/hydroxylapatite phantom used for the reproducibility tests was $188 \mathrm{mg}$. The average measured BM for the four film sets was $153.1 \pm 6.1 \mathrm{mg}$ (Table 2). The uncertainty is twice the SEM for the four sets of films. This gives a ratio between measured and weighed value of $0.814 \pm 0.032$.

For the linearity tests with the Perspex/hydroxylapatite phantoms, the ratio of measured $\mathrm{BM}$ to calcium hydroxylapatite mass is given by the slope of the line in Figure 5, that is $0.844 \pm 0.020$.

For the phantom used for the tests on thickness of overlying tissue, the measured $\mathrm{BM}(140.6 \mathrm{mg} \pm 7.7 \mathrm{mg})$ can be compared with the weighed value $169.0 \mathrm{mg}$ to give a ratio of $0.832 \pm 0.046$.

The average ratio for the three sets of measurements was $0.830 \pm 0.043$.

\section{Clinical Films}

In this work to date more than 700 pairs of $x$-ray images have been taken. Of these, approximately $9 \%$ could not be analyzed due to exposure, processing, or positioning faults. Results from 14 very-low-birth-weight infants are summarized here.

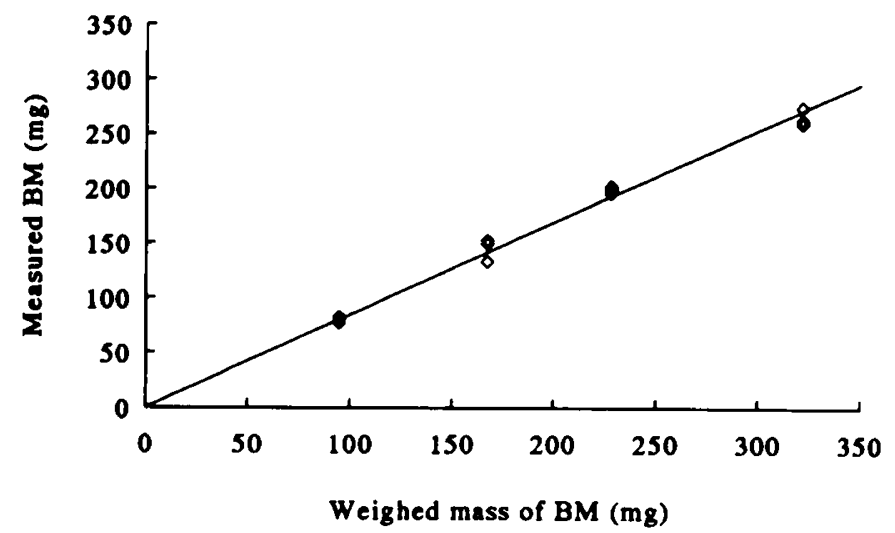

Figure 5. The total mass of calcium hydroxylapatite assessed by the absorptiometry technique (BM) plotted against the weighed mass in the Perspex/hydroxylapatite phantoms.
Table 3. Tests for the effect of the thickness of tissue overlying bone using a Perspex/hydroxylapatite phantom*

\begin{tabular}{rl}
\hline $\mathrm{t}(\mathrm{mm})$ & BM $(\mathrm{mg})$ \\
\hline 5.5 & $142.0 \pm 8.4$ \\
10.5 & $144.9 \pm 8.4$ \\
15.5 & $139.4 \pm 9.3$ \\
20.5 & $136.3 \pm 17.0$ \\
25.5 & $125.5 \pm 6.8$ \\
\hline
\end{tabular}

* The results are the average of three sets of measurements of BM with varying Perspex thicknesses ( $t$ ). The errors represent the $95 \%$ confidence limits.

The gestational ages ranged from 24 to $30 \mathrm{wk}$ (median $28 \mathrm{wk}$ ). The first analyzable radiograph for the babies in this group was taken between the first and 25th $d$ after birth, although only two babies did not have the initial measurement within the first week. It was noted that the $\mathrm{BM}$ within the radius changed slowly during the first $6 \mathrm{wk}$ of life and the BM at birth was calculated by linear extrapolation of the measured data in this initial phase to $\mathrm{d} 0$. The radius length was measured directly from the low-kV radiographs, and this could also be extrapolated back to age 0 because growth was linear. The BMC is the bone mineral per unit length, and these values were normalized to BMC at birth calculated from the extrapolated values of $\mathrm{BM}$ and length. The average value of $\mathrm{BMC}$ at birth was $2.84 \mathrm{mg} \cdot \mathrm{mm}^{-1}\left(\mathrm{SD}=0.60 \mathrm{mg} \cdot \mathrm{mm}^{-1}\right)$. The normalized data were averaged for the babies in the group and are presented in Figure 6.

\section{DISCUSSION}

A technique has been described for the measurement of bone mineral in the forearm of preterm infants that can be carried out on the ward with minimal disturbance. Suitable $x$-ray equipment for these measurements is available in most neonatal units. Additional equipment requirements are an arm holder including a double step wedge, a copper filter and filter holder for the high-kV film, and small-sized film cassettes that are not in routine use. Analysis of the images requires standard computational facilities with a TV camera, light box, and video digitizer. The cost of the additional equipment would be

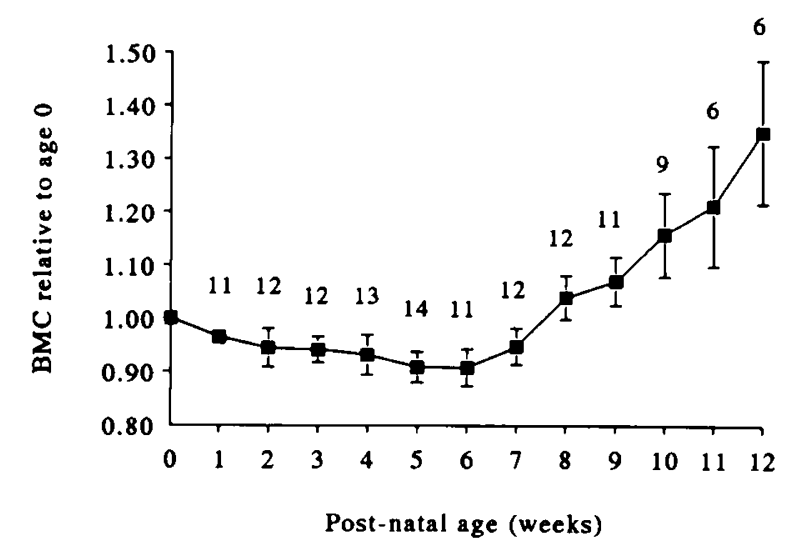

Figure 6. BMC normalized to birth plotted against postnatal age for a group of 14 preterm infants. 
approximately $£ 10000$, although suitable computing hardware may already be available in many imaging and medical physics departments. The technique could therefore be readily implemented elsewhere.

Tests have indicated a reproducibility of approximately $7 \%$, the main cause of variation being in the radiographic process. It has been shown that the results are linear up to at least $320 \mathrm{mg}$ of bone mineral distributed within an area of $4 \times 40 \mathrm{~mm}$. A systematic underestimate of bone mineral of approximately $17 \%$ has been observed. This has been reproducibly demonstrated with three phantom designs with films taken over a 2-y period of tests. No cause has yet been found for this.

The overlying thickness of soft tissue has not been found to affect the results up to an equivalent thickness of approximately $30 \mathrm{~mm}$. Above that level, some inaccuracy may be introduced because of the maximum thicknesses in the step wedge that is used for calibrating each film. In the analysis of the clinical films, no problems related to negative bone mineral values in the soft tissue background were noted. This indicates that the size limits set by the design of the step wedge were not encountered in the babies who were studied.

The results from infants reported here may be compared with results from the studies of Greer and McCormick (3) and Lyon et al. (5). Greer and McCormick, using a single energy photon absorptiometry technique, reported initial BMC of $3.18 \mathrm{mg} \cdot \mathrm{mm}^{-1}$ and $3.72 \mathrm{mg} \cdot \mathrm{mm}^{-1}$ for infants with 26- to 27-wk and 28- to 29-wk gestational ages, respectively. The mean value in the present study, if corrected for the systematic discrepancy noted above, was $3.39 \mathrm{mg} \cdot \mathrm{mm}^{-1}$ for a median gestational age of $28 \mathrm{wk}$. However, these data should not be compared directly, because Greer and McCormick measured at a single cross section of the radius at a distance of one third of the bone's length from its distal end. Lyon et al. studied infants with gestational ages between 25 and $30 \mathrm{wk}$ using a technique from which this work was developed. The initial BMC was reported to be $2.4 \mathrm{mg} \cdot \mathrm{mm}^{-1}$. However, this is for calcium plus phosphorus alone; the result, if corrected up to calcium hydroxylapatite, would be 4.11 $\mathrm{mg} \cdot \mathrm{mm}^{-1}$. They also reported longitudinal studies in which it was shown that BMC increased in the first week of life. This was not observed in this group of infants. They reported a subsequent decrease in BMC until age 6 wk, which has been confirmed in this report.

Certain improvements in the method are possible. In the work reported here, the time for the analysis of each film pair was approximately $20 \mathrm{~min}$. Subsequently, the software was transferred from the MicroVax computer to a SUN Sparcstation, a system that increasingly is used for imaging applications. This has reduced the analysis time to $4 \mathrm{~min}$. Improvements in the precision of measurement could be expected if a better imaging and digitization system were used. In part this might be achieved with a laser scanning film digitizer, which would eliminate the effect of light box inhomogeneity. However, this effect is the most predictable of the various inhomogeneities, and a significant improvement in precision would not be expected.

Most of the problems, other than those due to the heterogeneity of the $x$-ray beam itself, could be eliminated if a CR imaging system using photostimulable phosphor plates were used. However, such systems are very expensive and are currently available in very few hospitals. Our aim was to establish a technique in a hospital that does not have such facilities, using existing equipment with minimal additional cost. It is also important to note that present CR systems do not have an option for a plate size small enough to pass through the porthole of the incubator. The small film (detector) size that permits minimal disturbance to the infant is one of the major advantages of the system we have described, and this advantage would be lost if the technique were used in conjunction with CR technology.

A dual x-ray absorptiometry technique for the measurement of bone mineral in the forearm of preterm infants has been presented. It can be implemented in most neonatal units without the cost of additional expensive equipment. The technique involves minimal disturbance to the infant and has been used in this center for longitudinal studies that will be reported separately.

Acknowledgments. The authors thank Jean Williamson and the radiography staff of the Simpson Memorial Maternity Pavilion and the Royal Infirmary for their cooperation and assistance. We are grateful to Val Morgan and Breda McLaughlin for nursing assistance and the considerable help they have given toward the acquisition of the clinical data. We are particularly indebted to Martin Connell in the Department of Medical Physics for the development of the computer software used in this program.

\section{REFERENCES}

1. Mimouni F, Tsang RC 1987 Bone mineralization in infants: the contribution of photon absorptiometry. In: Stern L (ed) Feeding the Sick Infant, Nestlé Nutrition Workshop Series, Vol 11. Vevey/Raven Press, New York, pp 75-100

2. Tothill P 1989 Methods of bone mineral measurement. Phys Med Biol 34:543 572

3. Greer FR, McCormick A 1986 Bone growth with low bone mineral content in very low birth weight premature infants. Pediatr Res 20:925-928

4. Hawkes DJ, Doran M, McIntosh N, Lyon A, Theodoridou A, Williams JE 1989 Development of a digital radiographic technique to measure bone mineral in neonates. Calcif Tissue lnt 44:149

5. Lyon AJ, Hawkes DJ, Doran M. Mcintosh N, Chan F 1989 Bone mineralisation in preterm infants measured by dual energy radiographic densitometry. Arch Dis Child 64:919-923

6. Hawkes DJ, Jackson DF, Parker RP 1986 Tissue analysis by dual-energy computed tomography. Br J Radiol 59:537-542

7. Hubbell JH 1982 Photon mass attenuation and energy-absorption coefficients from $1 \mathrm{keV}$ to $20 \mathrm{MeV}$. Int J Appl Radiat Isot 33:1269-1290

8. Birch R, Marshall M, Ardran GM 1979 Catalogue of Spectral Data for Diagnostic X-rays, SRS 30. Hospital Physicists' Association, London

9. Hospital Physicists' Association 1977 Phantom Materials for Photons and Electrons, SRS 20. Hospital Physicists' Association, London 